\title{
Theory of Subcycle Linear Momentum Transfer in Strong-Field Tunneling Ionization
}

\author{
Hongcheng Ni®, ${ }^{1,2, *}$ Simon Brennecke, ${ }^{3}$ Xiang Gao $\odot,{ }^{2}$ Pei-Lun He, ${ }^{4, \dagger}$ Stefan Donsa, ${ }^{2}$ Iva Březinová, ${ }^{2}$ \\ Feng He, ${ }^{4}$ Jian Wu, ${ }^{1}$ Manfred Lein $\odot,{ }^{3}$ Xiao-Min Tong $\odot{ }^{5}$ and Joachim Burgdörfer, ${ }^{2, \$}$ \\ ${ }^{1}$ State Key Laboratory of Precision Spectroscopy, East China Normal University, Shanghai 200241, China \\ ${ }^{2}$ Institute for Theoretical Physics, Vienna University of Technology, 1040 Vienna, Austria, European Union \\ ${ }^{3}$ Institut für Theoretische Physik, Leibniz. Universität Hannover, 30167 Hannover, Germany, European Union \\ ${ }^{4}$ Key Laboratory for Laser Plasmas (Ministry of Education) and School of Physics and Astronomy, \\ Collaborative Innovation Center for IFSA (CICIFSA), Shanghai Jiao Tong University, Shanghai 200240, China \\ ${ }^{5}$ Center for Computational Sciences, University of Tsukuba, Tsukuba, Ibaraki 305-8573, Japan
}

(Received 19 April 2020; accepted 9 July 2020; published 10 August 2020)

\begin{abstract}
Interaction of a strong laser pulse with matter transfers not only energy but also linear momentum of the photons. Recent experimental advances have made it possible to detect the small amount of linear momentum delivered to the photoelectrons in strong-field ionization of atoms. We present numerical simulations as well as an analytical description of the subcycle phase (or time) resolved momentum transfer to an atom accessible by an attoclock protocol. We show that the light-field-induced momentum transfer is remarkably sensitive to properties of the ultrashort laser pulse such as its carrier-envelope phase and ellipticity. Moreover, we show that the subcycle-resolved linear momentum transfer can provide novel insights into the interplay between nonadiabatic and nondipole effects in strong-field ionization. This work paves the way towards the investigation of the so-far unexplored time-resolved nondipole nonadiabatic tunneling dynamics.
\end{abstract}

DOI: 10.1103/PhysRevLett.125.073202

Strong-field ionization is typically well described in the dipole approximation, in which the vector potential $\boldsymbol{A}$ of the electromagnetic field as well as the electric field $\boldsymbol{F}$ are assumed to be spatially homogeneous while the magnetic field vanishes. Consequently, also the Poynting vector vanishes. Thus, the strong field does not transfer linear momentum to the target. The dipole approximation is usually well justified for typical laser parameters employed in strong-field ionization in the so-called dipole oasis [1]. Remarkably, nondipole effects can be enhanced along two rather different routes-either by reducing the laser wavelength such that it approaches the atomic scale thereby probing the inhomogeneity of the electromagnetic field. This gives rise to a photoelectron angular distribution deviating from the dipole shape [2-7]. Alternatively, nondipole effects can also be enhanced by increasing the laser wavelength and/or increasing the intensity so that the motion of the liberated electron is strongly influenced by the magnetic field and radiation pressure of the laser field, resulting in linear momentum transfer along the propagation direction [8-18].

Published by the American Physical Society under the terms of the Creative Commons Attribution 4.0 International license. Further distribution of this work must maintain attribution to the author(s) and the published article's title, journal citation, and DOI.
Because of the photon dispersion relation $p=\mathcal{E} / c(c$ : the speed of light) included in the nondipole and relativistic regime [19-21], the momentum of a single photon in the propagation direction imparted on the target, $p_{z}$, is very small and is usually overshadowed by the transverse momentum $p_{\perp} \sim \sqrt{2 \mathcal{E}}$ gained by the electron from the photon energy $\mathcal{E}$, while other photon properties such as angular momentum [22-24] or helicity [25-27] have much more easily observable effects. With recent advances in detecting technologies, the small momentum shift $p_{z}$ in the laser propagation direction has become observable. In 2011, Smeenk et al. studied experimentally the sharing of the absorbed photon momentum between the photoelectron and the ion in tunneling ionization [8]. The photon momentum transfer could be viewed as a two-step process [15]. In the first step of tunneling, the electron and ion move together as a composite system, thus the ion gains almost all the photon momentum $I_{p} / c$ since it is much heavier than the electron $\left(I_{p}\right.$ : the ionization potential). In the second step of continuum motion, the liberated electron gains the momentum $E / c$, where $E$ is the final electron energy.

Further theoretical studies [28-30] found that the photon momentum transferred in the tunneling step is not given entirely to the ion due to the action of the laser magnetic field, resulting in the final electron momentum in the laser propagation direction 


$$
\left\langle p_{z}\right\rangle=E / c+\beta I_{p} / c
$$

where $\beta$ denotes the fraction of the momentum transferred to the electron during the tunneling step. Estimates for the ground-state hydrogen atom vary between $\beta=0.3[28,29]$ and $\beta=1 / 3[29,30]$.

While most previous studies focused on the momentum transfer by the entire pulse, very recently the first transverse-momentum-resolved study $[15,16]$ and angleresolved measurement of $\left\langle p_{z}\right\rangle$ [17] using an attoclock setup [31,32] became available. We present in the following the first $a b$ initio quantum simulation of the subcycle linear momentum transfer in strong-field ionization resolved in time. We refer to this process as light-field-induced momentum transfer since effects of quantization of the radiation field, i.e., photonic properties, play no significant role in the strong-field regime. We show that the subcycleresolved momentum transfer $\left\langle p_{z}\left(\phi_{p}\right)\right\rangle\left(\phi_{p}\right.$ is the attoclock angle in the polarization plane, Fig. 1) sensitively depends on the optical properties of the ultrashort pulse, most notably its carrier-envelope phase (CEP) and polarization. Conceptually importantly, it provides novel insights into the momentum sharing between the departing electron and the residual ion. Employing the backpropagation method [33-35], we are able to separate the longitudinal momentum transferred during the tunneling process and during the continuum motion of the liberated electron on a subcycle scale. Moreover, we find the time-resolved momentum transfer to be sensitive to the temporal variation of the tunneling barrier, i.e., to nonadiabatic tunneling effects. Nonadiabaticity has been known to induce energy variations across the tunneling barrier (total energy at the tunnel exit different from the initial-state energy) and shifts of the

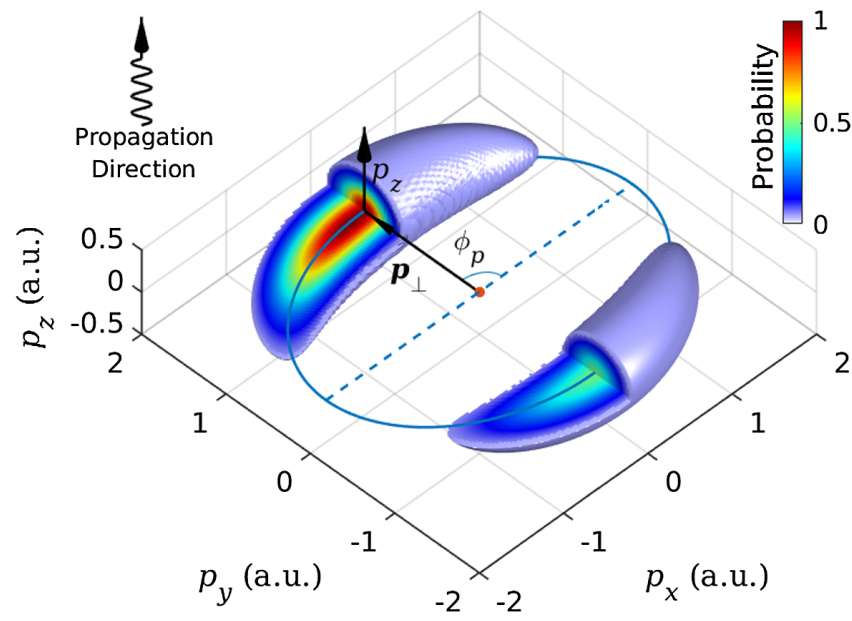

FIG. 1. Attoclock protocol for subcycle-resolved electron emission with momentum $\boldsymbol{p}=\left(\boldsymbol{p}_{\perp}, p_{z}\right)$ with the longitudinal component $p_{z}$ along the propagation direction and the transverse component $\boldsymbol{p}_{\perp}$ in the polarization plane. For a few-cycle pulse with ellipticity $\varepsilon$, the classical cycle-averaged radiation pressure picture suggests $\left\langle p_{z}\right\rangle>0$. central transverse tunneling momentum from zero [34-37]. Here, we identify modulations in the linear momentum transfer, both at the tunnel exit and in the asymptotic regime, stemming from the nonadiabatic tunneling dynamics, demonstrating an interplay between the nonadiabatic and nondipole tunneling effects.

We numerically solve the time-dependent Schrödinger equation (TDSE) for strong-field ionization of helium with the single-active-electron approximation beyond the dipole approximation by including corrections to first order in $1 / c$ (in atomic units). The Hamiltonian is given by $[15,38]$

$$
\begin{aligned}
H= & \frac{1}{2}\left[\boldsymbol{p}+\boldsymbol{A}(t)+\frac{\boldsymbol{e}_{z}}{c}\left(\boldsymbol{p} \cdot \boldsymbol{A}(t)+\frac{1}{2} A^{2}(t)\right)\right]^{2} \\
& +V\left(\boldsymbol{r}-\frac{z}{c} \boldsymbol{A}(t)\right)
\end{aligned}
$$

where $(x, y)$ is the laser polarization plane (denoted by the subscript $\perp$ ) and $\hat{z}$ is along the direction of the laser propagation and longitudinal momentum transfer (Fig. 1), $\boldsymbol{p}$ is the momentum operator, $\boldsymbol{r}$ is the position operator, $V(\boldsymbol{r})$ is the effective potential for the helium atom [39], and $\boldsymbol{A}(t)=\boldsymbol{A}(t, z=0)$ is the laser vector potential at the position of the nucleus. In the following, we choose $\boldsymbol{A}(t)=$ $A_{0} \cos ^{4}(\omega t / 2 N)\left[\sin \left(\omega t+\phi_{\mathrm{CEP}}\right) \boldsymbol{e}_{x}-\varepsilon \cos \left(\omega t+\phi_{\mathrm{CEP}}\right) \boldsymbol{e}_{y}\right]$, where $A_{0}$ is the peak amplitude, $\varepsilon$ is the ellipticity, $\omega$ is the central angular frequency, $N$ is the total number of cycles, and $\phi_{\text {CEP }}$ is the CEP. The corresponding electric field is defined as $\boldsymbol{F}(t)=-\dot{\boldsymbol{A}}(t)$. We use a laser pulse with a wavelength of $\lambda=800 \mathrm{~nm}$, a total intensity of $5 \times 10^{14} \mathrm{~W} / \mathrm{cm}^{2}, N=6, \varepsilon=0.75$, and $\phi_{\mathrm{CEP}}=0$ or $\pi / 2$, unless specified otherwise. For these laser parameters, the Keldysh parameter amounts to 0.80 , corresponding to ionization in the near tunneling regime. Since a short laser pulse is used, the ponderomotive gradient from the laser focus does not transfer any net linear momentum to the electron [8]. We note that nondipole effects will increase in the midinfrared with $\lambda^{2}$ (see Ref. [40]). We demonstrate here that they are already observable in the near infrared. The TDSE is solved by two alternative methods to check for convergence. We use the split-operator Fourier method on a grid with 1024 points in each dimension, a grid step of 0.35 a.u., and a time step of 0.03 a.u. The simulation box is separated smoothly into an inner and an outer part by an absorbing boundary of the form $1 /\left[1+\exp \left\{\left(r-r_{0}\right) / d\right\}\right]$, where $r_{0}=164$ and $d=4$ a.u. The inner part is propagated using the full Hamiltonian and the outer part using a Coulomb-free Hamiltonian. At each time step, the absorbed wave function is projected onto the nondipole Volkov solution $[29,53]$ to incrementally obtain the momentum distribution $[54,55]$. Alternatively, the TDSE is also solved with the generalized pseudospectral method [56-58], where the time propagation of the nondipole contribution is treated using a Taylor expansion in combination with a split-operator method [40]. We have verified that the two 
numerical methods give virtually identical results. As an additional tool we employ the backpropagation method [33-35]. It allows us to extract from the full TDSE result for the flux histogram or final wave function the local characteristics including the momentum distribution at the tunnel exit. While not directly an experimental observable itself, it can provide additional key insights into the underlying tunneling dynamics. To this end, we place a sphere of 6000 evenly distributed virtual detectors [59-64] at a radius $r_{\mathrm{vd}}=40$ a.u. to convert the quantum flux into an ensemble of classical trajectories [40], which are subsequently propagated backward in time to retrieve the tunneling exit characteristics [26,33-35]. $r_{\mathrm{vd}}$ is chosen such that no unphysical intercycle interference distorts the backpropagation thereby protecting the phase gradient as the local momentum [59-62]. We have checked that the results do not depend on the particular choice of $r_{\mathrm{vd}}$ and are converged with respect to the number of virtual detectors used. The wave function is propagated for an additional cycle period after the pulse conclusion such that most ionized electronic flux reached the detector sphere.

In order to explore analytically the partitioning of the light-field-induced momentum transfer between electron and ion in tunneling ionization in the subcycle regime, we extend the strong-field approximation (SFA) to include both nondipole and nonadiabatic effects simultaneously (ndSFA). Accordingly, after applying the stationary-phase approximation, the tunneling-ionization rate is given by [65-67]

$$
W_{\text {ndSFA }}=|\ddot{S}|^{-\alpha_{Z}} \exp \{2 \operatorname{Im} S\}
$$

with $\alpha_{Z}=1+Z / \sqrt{2 I_{p}}$ ( $Z$ is the asymptotic charge of the remaining ion) and $S=\int_{t_{s}}^{t_{r}}\left(\frac{1}{2}\left\{\boldsymbol{p}+\boldsymbol{A}(t)+\left(\boldsymbol{e}_{z} / c\right)[\boldsymbol{p}\right.\right.$. $\left.\left.\left.\boldsymbol{A}(t)+\frac{1}{2} A^{2}(t)\right]\right\}^{2}+I_{p}\right) d t[29,53]$ evaluated at the complex saddle-point time $t_{s}=t_{r}+i t_{i}$, where the real part $t_{r}$ denotes the time the electron exits the tunneling barrier and the imaginary part $t_{i}$ is related to the tunnelingionization probability. Equation (3) includes nonadiabatic as well as nondipole effects and keeps track of nonexponential prefactors. Remarkably, all of these factors leave their signature on the subcycle-resolved longitudinal momentum transfer $\left\langle p_{z}\right\rangle$. The present ndSFA description allows us to disentangle the longitudinal momentum transfer during tunneling from that of the free motion after tunneling with subcycle resolution.

The initial kinetic momentum at the tunnel exit $v$ is related to the final momentum $\boldsymbol{p}$ by $\boldsymbol{v}=\boldsymbol{p}+\boldsymbol{A}+\left(\boldsymbol{e}_{z} /\right.$ $2 c)\left[\left(\boldsymbol{p}_{\perp}+\boldsymbol{A}\right)^{2}-p_{\perp}^{2}\right]=\boldsymbol{p}+\boldsymbol{A}+\left(\boldsymbol{e}_{z} / 2 c\right)\left(v_{\perp}^{2}-p_{\perp}^{2}\right)$. The ndSFA prediction for $v$ can be tested against the numerical results from the backpropagation of the full TDSE solution. The time-resolved longitudinal momentum at the tunnel exit $\left\langle v_{z}\left(t_{r}\right)\right\rangle$ is found to be approximately given by [see Ref. [40], Eq. (S29)]

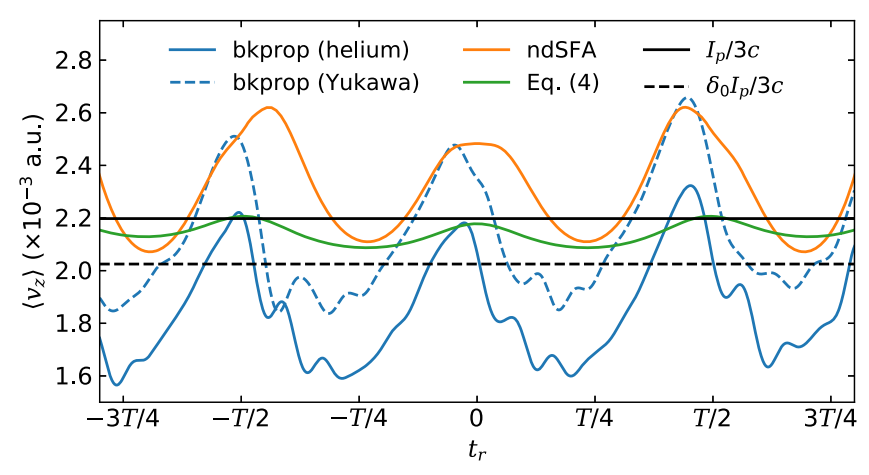

FIG. 2. Subcycle time-resolved linear momentum transfer at the tunnel exit for a sinelike pulse $\phi_{\mathrm{CEP}}=\pi / 2$. Blue curve: backpropagation of TDSE wave packet (bkprop) for the helium potential (solid curve) and the short-range Yukawa-like potential (dashed curve); orange curve: ndSFA using $\alpha_{0}=1$; green curve: analytical prediction for $\left\langle v_{z}\right\rangle$ [Eq. (4)] using $\alpha_{0}=1$; black solid curve: $I_{p} / 3 c$; black dashed curve: $\delta_{0} I_{p} / 3 c$, with $\delta_{0}=1-2 \alpha_{0} F_{0} /\left(2 I_{p}\right)^{3 / 2}$.

$$
\left\langle v_{z}\left(t_{r}\right)\right\rangle=\frac{\tilde{I_{p}}\left(t_{r}\right)}{3 c}\left[1-\frac{2 \alpha_{Z} F\left(t_{r}\right)}{\left(2 I_{p}\right)^{3 / 2}}\right],
$$

with an effective ionization potential $\tilde{I}_{p}\left(t_{r}\right) \equiv I_{p}+$ $\left\langle v_{\perp}^{2}\left(t_{r}\right)\right\rangle / 2$ accounting for the energy shift by the transverse tunneling momentum. The correction term proportional to $F$ approximately incorporates the influence from the nonexponential prefactor. The asymptotic longitudinal momentum $\left\langle p_{z}\left(t_{r}\right)\right\rangle$ follows as $[16,40]$

$$
\left\langle p_{z}\left(t_{r}\right)\right\rangle=\Delta E / c+\left\langle v_{z}\left(t_{r}\right)\right\rangle,
$$

providing for the subcycle decomposition of the light-fieldinduced longitudinal momentum transfer into one part associated with the tunneling dynamics $\left\langle v_{z}\right\rangle$ and another associated with the free-particle motion of the liberated electron [68] with $\Delta E=\frac{1}{2}\left(\left\langle p_{\perp}^{2}\right\rangle-\left\langle v_{\perp}^{2}\right\rangle\right)$. For $\left\langle v_{\perp}^{2}\right\rangle \ll$ $\left\langle p_{\perp}^{2}\right\rangle$ and $\alpha_{Z}=0$, Eq. (5) resembles the cycle-averaged limit [Eq. (1)]. However, significant modifications appear. Only the energy gained in the streaking field after ionization, $\Delta E$, rather than the total energy $E$, contributes to the first term $[15,16]$. More importantly, the longitudinal momentum transfer during tunneling $\left\langle v_{z}\left(t_{r}\right)\right\rangle$ displays a $2 \omega$ subcycle oscillation and additionally a downward shift relative to the value $I_{p} / 3 c$ (Fig. 2). Scrutinizing Eq. (4), we can identify different contributions: neglecting $\left\langle v_{\perp}^{2}\right\rangle$ and the preexponential prefactor $\left(\alpha_{Z}=0\right)$, Eq. (4) reduces to $\left\langle v_{z}\right\rangle=I_{p} / 3 c$ (black solid line). Including the prefactor for a short-range potential $\left(\alpha_{0}=1\right)$, the $t_{r}$-independent part is shifted downward to $\left\langle v_{z}\right\rangle=I_{p} / 3 c\left[1-2 \alpha_{0} F_{0} /\left(2 I_{p}\right)^{3 / 2}\right]$ (black dashed line), with the prefactor evaluated at the field peak $F_{0}=A_{0} \omega$, where the ionization probability maximizes. It approximately agrees with the minima for the backpropagated wave packet in a Yukawa-like potential $V(r)=-\left[1.17822 \exp (-0.2 r)+\exp \left(-0.5 r^{2}\right)\right] /$ $\sqrt{r^{2}+0.14328}$ with the same $I_{p}$ as helium (blue dashed 
curve). On top of this downward shift, nonadiabaticity contributes to an upward shift $\sim\left\langle v_{\perp}^{2}\left(t_{r}\right)\right\rangle$ featuring a $2 \omega$ subcycle oscillation reproduced by both Eq. (4) and ndSFA, where the smaller oscillation amplitude of Eq. (4) is primarily due to the expansion in $t_{i}$ [40]. The backpropagation from the full helium potential (blue solid curve) reveals an additional downward shift due to the presence of Coulomb attraction during the underbarrier motion of the ionizing electron.

The subcycle variation of the ionization probability as well as of the light-field-induced momentum transfer is found to strongly depend on the CEP of the pulse (Fig. 3) shown for a cosinelike pulse ( $\phi_{\mathrm{CEP}}=0$, left column) and a sinelike pulse ( $\phi_{\mathrm{CEP}}=\pi / 2$, right column). The tunnelingionization rates obtained by the ndSFA reproduce (up to a scaling factor) quite well the rates determined by the full quantum TDSE forward propagation followed by the semiclassical backward propagation [Fig. 3(a)]. While the cosinelike pulse yields a single dominant peak at the
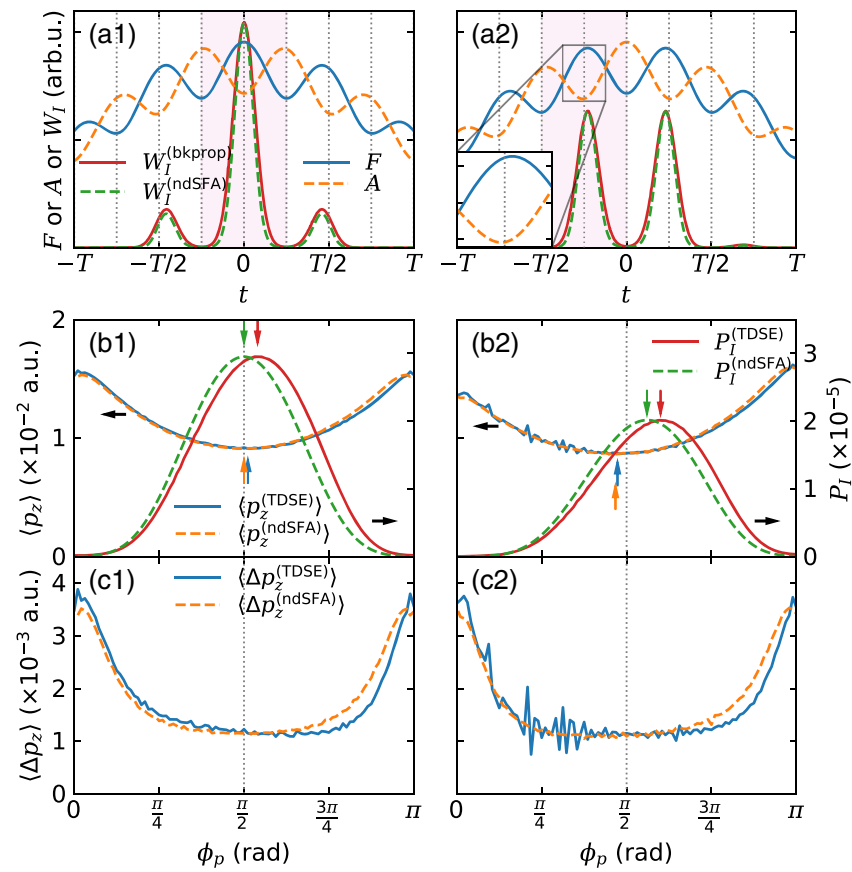

FIG. 3. Row (a): Absolute magnitude of the electric field $F(t)$ and vector potential $A(t)$ of the ultrashort few-cycle pulse with $\phi_{\mathrm{CEP}}=0$ (left column) and $\phi_{\mathrm{CEP}}=\pi / 2$ (right column). Also shown is the tunneling ionization rate $W_{I}\left(t=t_{r}\right)$ calculated by backpropagation (bkprop) and ndSFA using $\alpha_{1}=1+1 / \sqrt{2 I_{p}}$ as a function of the tunneling exit time $t_{r}$. Row (b): Asymptotic longitudinal momentum transfer $\left\langle p_{z}\left(\phi_{p}\right)\right\rangle$ (left ordinate) and ionization probability $P_{I}\left(\phi_{p}\right)$ (right ordinate) as a function of the attoclock angle $\phi_{p}$ calculated by TDSE and ndSFA for the attoclock signal corresponding to the shaded temporal half cycle in row (a). The arrows mark the phases of the maxima of $P_{I}$ and minima of $\left\langle p_{z}\right\rangle$. Row (c): Nonadiabatic longitudinal momentum transfer $\left\langle\Delta p_{z}\left(\phi_{p}\right)\right\rangle$ as a function of the attoclock angle $\phi_{p}$ calculated by TDSE and ndSFA. pulse center $t=0$ (left column), the sinelike pulse gives rise to two peaks of comparable magnitude (right column). Note that for the few-cycle elliptic pulse, the phase shift between the peaks in the $A(t)$ and the $F(t)$ fields deviates from $\pi / 2$ and becomes itself time dependent [Fig. 3(a)]. Within the attoclock setting, the asymptotic momentum distribution projected onto the polarization plane provides information on the subcycle phase (or attoclock angle), $\phi_{p}=\arctan \left(p_{y} / p_{x}\right)$. This phase can be mapped onto the phase of the electromagnetic field through the attoclock (or streaking) principle $\boldsymbol{p}_{\perp} \approx-\boldsymbol{A}$ thereby allowing us to extract subcycle timing information from the rotating $\boldsymbol{A}$ vector. The peak position of the ionization probability $P_{I}\left(\phi_{p}\right)$ is phase shifted between the full TDSE solution and the ndSFA [Fig. 3(b)] which is a well-known signature of Coulomb scattering of the outgoing electron neglected in the ndSFA. More surprisingly, the peak position of $P_{I}\left(\phi_{p}\right)$ varies strongly with $\phi_{\text {CEP }}$ [Fig. 3(b)]. In fact, this variation, virtually identical for both TDSE and ndSFA, is significantly larger than the Coulomb-field-induced shift. The momentum distribution $P\left(\phi_{p}, p_{z}\right)$ (Fig. 1) features a slight asymmetry in $p_{z}$ representing a signature of nondipole effects. In most previous studies $[8,10,14,18,28-30]$, the mean shift in $p_{z}$ has been examined, i.e., $\left\langle p_{z}\right\rangle=$ $\int d \phi_{p} d p_{z} p_{z} P\left(\phi_{p}, p_{z}\right) / \int d \phi_{p} d p_{z} P\left(\phi_{p}, p_{z}\right)$. We resolve the shift in $p_{z}$ at a given attoclock angle, i.e., $\left\langle p_{z}\left(\phi_{p}\right)\right\rangle=$ $\int d p_{z} p_{z} P\left(\phi_{p}, p_{z}\right) / \int d p_{z} P\left(\phi_{p}, p_{z}\right)$ [Fig. 3(b)]. The subcycle dependence of $\left\langle p_{z}\left(\phi_{p}\right)\right\rangle$, predicted by TDSE is well reproduced by ndSFA [Eq. (3)] despite ndSFA neglects the Coulomb interaction of the outgoing electron with the residual ion. The Coulomb interaction is, thus, not important for the asymptotic longitudinal momentum transfer, because, to leading order, Coulomb-laser coupling is absent as there is no laser field in the propagation direction [16]. We note that, for linear polarization, the Coulomb effect plays a much more important role in the asymptotic longitudinal momentum transfer, both for single [9-11, 14] and double ionization [18].

For ellipticities well below $\varepsilon=1,\left\langle p_{z}\right\rangle$ features a pronounced minimum near $\phi_{p} \approx \pi / 2$ (for details see Ref. [40]). This follows directly from Eq. (5). The energy imparted by the laser field is approximately given by $\Delta E=\left(\left\langle p_{\perp}^{2}\right\rangle-\left\langle v_{\perp}^{2}\right\rangle\right) / 2 \approx A^{2} / 2$. Since the magnitude of the electric field $F(t)$ reaches its maximum near $\phi_{p} \approx \pi / 2$, $A(t)$ reaches a local minimum at this angle which, in turn, translates into a minimum of $\left\langle p_{z}\right\rangle$. The precise position of this minimum $\phi_{p}\left(p_{z}^{\mathrm{min}}\right)$ depends, however, sensitively on the CEP. While for a cosinelike pulse $\left(\phi_{\mathrm{CEP}}=0\right), \phi_{p}\left(p_{z}^{\min }\right)$ is located at $\phi_{p}=\pi / 2$, for a sinelike pulse $\left(\phi_{\mathrm{CEP}}=\pi / 2\right)$, the minimum is shifted to smaller $\phi_{p}$ [Fig. 3(b)]. Also this variation can be understood as a consequence of Eq. (5): for $\phi_{\mathrm{CEP}}=0$, the maximum of $F$ coincides with the minimum of $A$ [Fig. 3(a1)]; for $\phi_{\mathrm{CEP}}=\pi / 2$, the maximum of $F$ and the minimum of $A$ are slightly displaced from each other from the expected instance of time at $\omega t= \pm \pi / 2$ [inset 
Fig. 3(a2)] resulting in the observed phase shift. Such subcycle modulation of the linear momentum transfer will survive focal volume averaging since this timing is independent of the laser intensity [40].

Of considerable conceptual interest is now to what extent nonadiabatic tunneling effects may leave their mark on the experimentally observable asymptotic linear momentum transfer $\left\langle p_{z}\right\rangle$. Since $\left\langle p_{z}\left(t_{r}\right)\right\rangle=A^{2}\left(t_{r}\right) / 2 c-$ $\left\langle\boldsymbol{v}_{\perp}\left(t_{r}\right)\right\rangle \cdot \boldsymbol{A}\left(t_{r}\right) / c+\left\langle v_{z}\left(t_{r}\right)\right\rangle$ [Eq. (5)], the nonadiabaticity-induced subcycle variation in $\left\langle v_{\perp}\left(t_{r}\right)\right\rangle$ not only leads to modulations of the linear momentum transfer at the tunnel exit but also is amplified in $\left\langle p_{z}\right\rangle$ by its coupling to the vector potential. Consequently, when subtracting from $\left\langle p_{z}\right\rangle$ the contributions present in the adiabatic limit, $\left\langle\Delta p_{z}\right\rangle=$ $\left\langle p_{z}\right\rangle-A^{2} / 2 c-I_{p} / 3 c$, we find a pronounced angular modulation [Fig. 3(c)] of the residual signal that is independent of the laser CEP which should facilitate its detection [40]. Moreover, the nonadiabatic tunneling correction also leads to an overall increase in $\left\langle p_{z}\right\rangle$ since $\left\langle\boldsymbol{v}_{\perp}\left(t_{r}\right)\right\rangle \cdot \boldsymbol{A}\left(t_{r}\right)<0$.

Both the angle of the minimum of $\left\langle p_{z}\left(\phi_{p}\right)\right\rangle, \phi_{p}\left(p_{z}^{\min }\right)$, and the angle of the maximum of $P_{I}\left(\phi_{p}\right), \phi_{p}\left(P_{I}^{\max }\right)$, feature a systematic variation as a function of the ellipticity and the CEP [Figs. 4(a) and 4(b)]. Here, we focus on the first attoclock ionization peaks $\left[0<\phi_{p}<\pi\right.$, shaded area in Fig. 3(a)]. The value of $\phi_{p}\left(p_{z}^{\min }\right)$ for a sinelike $\left(\phi_{\mathrm{CEP}}=\pi / 2\right)$ and a $(-1)$ sinelike pulse $\left(\phi_{\mathrm{CEP}}=-\pi / 2\right)$ differ from each other [Fig. 4(a)]. The light-field-induced longitudinal momentum $\left\langle p_{z}\left(\phi_{p}\right)\right\rangle$ for an ultrashort pulse is a manifestly non-inversion-symmetric observable. For elliptically polarized pulses, the phase shift $\Delta \phi_{p}$ between the minimum of $\left\langle p_{z}\right\rangle$ and the maximum of $P_{I}$, $\Delta \phi_{p}=\phi_{p}\left(P_{I}^{\max }\right)-\phi_{p}\left(p_{z}^{\min }\right)$, can be directly converted into a time delay $\Delta \tau=\Delta \phi_{p} / \omega$ [Fig. 4(c)]. We find time delays of the order of tens of attoseconds. Such time delays have indeed been observed recently, however without CEP
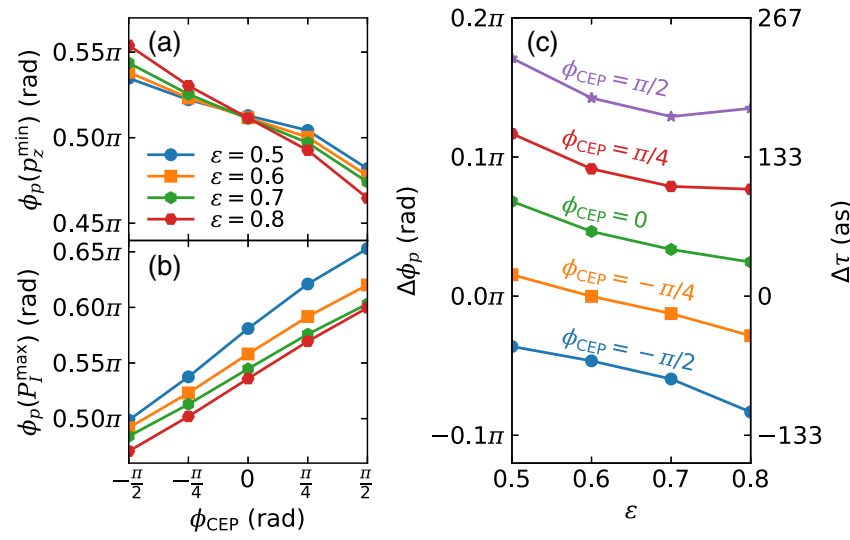

FIG. 4. The dependence of the attoclock angle $\phi_{p}\left(p_{z}^{\min }\right)$ (a) and $\phi_{p}\left(P_{I}^{\max }\right)(\mathrm{b})$ on the CEP for different ellipticities. The attoclock angle differences $\Delta \phi_{p}$ between $\phi_{p}\left(p_{z}^{\min }\right)$ and $\phi_{p}\left(P_{I}^{\max }\right)$ as a function of ellipticity $\varepsilon$ for different CEP's (c) (left ordinate) can be converted to a time delay $\Delta \tau$ in attoseconds (right ordinate). control [17]. In view of the strong CEP dependence revealed by our simulation [Fig. 4(c)], the interpretation of this delay has remained inconclusive. In turn, future CEP-resolved measurements of $\Delta \tau$ would offer probes of the subcycle timing of the momentum transfer in unprecedented detail.

In summary, we have simulated the subcycle timeresolved light-field-induced linear momentum transfer beyond dipole approximation during strong-field tunneling ionization employing an attoclock protocol. A pronounced minimum in the momentum transfer is found along the minor axis of the polarization ellipse where the ionization probability peaks. The attoclock phase angle or timing of the minimum in the momentum transfer is shown to be strongly dependent on the carrier-envelope phase. By comparison between the backpropagation of the full TDSE solution to the tunnel exit and a novel strong-field approximation including nondipole and nonadiabatic effects, distinct contributions to the light-field-induced momentum transfer on a subcycle scale could be identified. The mean momentum shift is found to be sensitive to subexponential as well as Coulomb contributions to tunneling. Nonadiabatic corrections result in a $2 \omega$ subcycle modulation of the transferred momentum at the tunnel exit and in the asymptotic region. Such nonadiabaticity-induced modulations may become directly observable in future experiments. The would represent the first direct evidence of an interplay between nonadiabatic and nondipole tunneling effects. The longitudinal momentum transfer thus promises to offer new insights into the strong-field tunneling dynamics.

This work was supported by Projects No. 11904103, No. 11774023, and No. 11574205 of the National Natural Science Foundation of China (NSFC), Projects No. M2692 and No. W1243 of the Austrian Science Fund (FWF), the Programme Quantum Dynamics in Tailored Intense Fields (QUTIF) of the Deutsche Forschungsgemeinschaft (DFG), Project No. MA14-002 of the Vienna Science and Technology Fund (WWTF), Project No. 19JC1412200 of the Shanghai Science and Technology Commission, the Fundamental Research Funds for the Central Universities, Projects No. 2018YFA0404802 and No. 2018YFA0306303 of the National Key R\&D Program of China, and Project No. JP16K05495 of the Grant-in-Aid for Scientific Research of the Japan Society for the Promotion of Science. Numerical simulations were in part performed on the Vienna Scientific Cluster (VSC).

*hcni@1ps.ecnu.edu.cn †a225633@sjtu.edu.cn \#joachim.burgdoerfer@tuwien.ac.at

[1] H. R. Reiss, The tunnelling model of laser-induced ionization and its failure at low frequencies, J. Phys. B 47, 204006 (2014). 
[2] O. Hemmers, R. Guillemin, and D. W. Lindle, Nondipole effects in soft X-ray photoemission, Radiat. Phys. Chem. 70, 123 (2004).

[3] B. Krässig, E. P. Kanter, S. H. Southworth, R. Guillemin, O. Hemmers, D. W. Lindle, R. Wehlitz, and N. L. S. Martin, Photoexcitation of a Dipole-Forbidden Resonance in Helium, Phys. Rev. Lett. 88, 203002 (2002).

[4] M.-X. Wang, X.-R. Xiao, H. Liang, S.-G. Chen, and L.-Y. Peng, Photon-momentum transfer in one- and two-photon ionization of atoms, Phys. Rev. A 96, 043414 (2017).

[5] S. Grundmann, F. Trinter, A. W. Bray, S. Eckart, J. Rist, G. Kastirke, D. Metz, S. Klumpp, J. Viefhaus, L. P. H. Schmidt, J. B. Williams, R. Dörner, T. Jahnke, M. S. Schöffler, and A. S. Kheifets, Separating Dipole and Quadrupole Contributions to Single-Photon Double Ionization, Phys. Rev. Lett. 121, 173003 (2018).

[6] S. Grundmann, M. Kircher, I. Vela-Perez, G. Nalin, D. Trabert, N. Anders, N. Melzer, J. Rist, A. Pier, N. Strenger, J. Siebert, P. V. Demekhin, L. P. H. Schmidt, F. Trinter, M. S. Schöffler, T. Jahnke, and R. Dörner, Observation of Photoion Backward Emission in Photoionization of $\mathrm{He}$ and $\mathrm{N}_{2}$, Phys. Rev. Lett. 124, 233201 (2020).

[7] S.-G. Chen, W.-C. Jiang, S. Grundmann, F. Trinter, M. S. Schöffler, T. Jahnke, R. Dörner, H. Liang, M.-X. Wang, L.-Y. Peng, and Q. Gong, Photon Momentum Transfer in Single-Photon Double Ionization of Helium, Phys. Rev. Lett. 124, 043201 (2020).

[8] C. T. L. Smeenk, L. Arissian, B. Zhou, A. Mysyrowicz, D. M. Villeneuve, A. Staudte, and P. B. Corkum, Partitioning of the Linear Photon Momentum in Multiphoton Ionization, Phys. Rev. Lett. 106, 193002 (2011).

[9] J. Liu, Q. Z. Xia, J. F. Tao, and L. B. Fu, Coulomb effects in photon-momentum partitioning during atomic ionization by intense linearly polarized light, Phys. Rev. A 87, 041403(R) (2013).

[10] A. Ludwig, J. Maurer, B. W. Mayer, C. R. Phillips, L. Gallmann, and U. Keller, Breakdown of the Dipole Approximation in Strong-Field Ionization, Phys. Rev. Lett. 113, 243001 (2014).

[11] J. F. Tao, Q. Z. Xia, J. Cai, L. B. Fu, and J. Liu, Coulomb rescattering in nondipole interaction of atoms with intense laser fields, Phys. Rev. A 95, 011402(R) (2017).

[12] J. Maurer, B. Willenberg, J. Daněk, B. W. Mayer, C. R. Phillips, L. Gallmann, M. Klaiber, K. Z. Hatsagortsyan, C. H. Keitel, and U. Keller, Probing the ionization wave packet and recollision dynamics with an elliptically polarized strong laser field in the nondipole regime, Phys. Rev. A 97, 013404 (2018).

[13] B. Willenberg, J. Maurer, U. Keller, J. Daněk, M. Klaiber, N. Teeny, K. Z. Hatsagortsyan, and C. H. Keitel, Holographic interferences in strong-field ionization beyond the dipole approximation: The influence of the peak and focal-volumeaveraged laser intensities, Phys. Rev. A 100, 033417 (2019).

[14] N. Haram, I. Ivanov, H. Xu, K. T. Kim, A. Atia-tul-Noor, U. S. Sainadh, R. D. Glover, D. Chetty, I. V. Litvinyuk, and R. T. Sang, Relativistic Nondipole Effects in Strong-Field Atomic Ionization at Moderate Intensities, Phys. Rev. Lett. 123, 093201 (2019).

[15] A. Hartung, S. Eckart, S. Brennecke, J. Rist, D. Trabert, K. Fehre, M. Richter, H. Sann, S. Zeller, K. Henrichs,
G. Kastirke, J. Hoehl, A. Kalinin, M. S. Schöffler, T. Jahnke, L. P. H. Schmidt, M. Lein, M. Kunitski, and R. Dörner, Magnetic fields alter strong-field ionization, Nat. Phys. 15, 1222 (2019).

[16] N. Eicke, S. Brennecke, and M. Lein, Attosecond-Scale Streaking Methods for Strong-Field Ionization by Tailored Fields, Phys. Rev. Lett. 124, 043202 (2020).

[17] B. Willenberg, J. Maurer, B. W. Mayer, and U. Keller, Subcycle time resolution of multi-photon momentum transfer in strong-field ionization, Nat. Commun. 10, 5548 (2019).

[18] F. Sun, X. Chen, W. Zhang, J. Qiang, H. Li, P. Lu, X. Gong, Q. Ji, K. Lin, H. Li, J. Tong, F. Chen, C. Ruiz, J. Wu, and F. He, Longitudinal photon-momentum transfer in strong-field double ionization of argon atoms, Phys. Rev. A 101, 021402 (R) (2020).

[19] V. D. Mur, B. M. Karnakov, and V. S. Popov, Relativistic version of the imaginary-time formalism, J. Exp. Theor. Phys. 87, 433 (1998).

[20] V.S. Popov, B. M. Karnakov, and V. D. Mur, On the relativistic theory of tunneling, JETP Lett. 79, 262 (2004).

[21] V. S. Popov, B. M. Karnakov, V. D. Mur, and S. G. Pozdnyakov, Relativistic theory of tunnel and multiphoton ionization of atoms in a strong laser field, J. Exp. Theor. Phys. 102, 760 (2006).

[22] F. Maulbetsch and J. Briggs, Selection rules for transitions to two-electron continuum states, J. Phys. B 28, 551 (1995).

[23] H. Ni, S. Chen, C. Ruiz, and A. Becker, Selection rules in the few-photon double ionization of the helium atom, J. Phys. B 44, 175601 (2011).

[24] K. Henrichs, S. Eckart, A. Hartung, D. Trabert, J. Rist, H. Sann, M. Pitzer, M. Richter, H. Kang, M. S. Schöffler, M. Kunitski, T. Jahnke, and R. Dörner, Experimental evidence for selection rules in multiphoton double ionization of helium and neon,Phys. Rev. A 97, 031405(R) (2018).

[25] I. Barth and O. Smirnova, Nonadiabatic tunneling in circularly polarized laser fields: Physical picture and calculations, Phys. Rev. A 84, 063415 (2011).

[26] K. Liu, H. Ni, K. Renziehausen, J. M. Rost, and I. Barth, Deformation of Atomic $p_{ \pm}$Orbitals in Strong Elliptically Polarized Laser Fields: Ionization Time Drifts and Spatial Photoelectron Separation, Phys. Rev. Lett. 121, 203201 (2018).

[27] S. Eckart, M. Kunitski, M. Richter, A. Hartung, J. Rist, F. Trinter, K. Fehre, N. Schlott, K. Henrichs, L. P. H. Schmidt, T. Jahnke, M. Schöffler, K. Liu, I. Barth, J. Kaushal, F. Morales, M. Ivanov, O. Smirnova, and R. Dörner, Ultrafast preparation and detection of ring currents in single atoms, Nat. Phys. 14, 701 (2018).

[28] S. Chelkowski, A. D. Bandrauk, and P. B. Corkum, Photon Momentum Sharing between an Electron and an Ion in Photoionization: From One-Photon (Photoelectric Effect) to Multiphoton Absorption, Phys. Rev. Lett. 113, 263005 (2014).

[29] P.-L. He, D. Lao, and F. He, Strong Field Theories beyond Dipole Approximations in Nonrelativistic Regimes, Phys. Rev. Lett. 118, 163203 (2017).

[30] M. Klaiber, E. Yakaboylu, H. Bauke, K. Z. Hatsagortsyan, and C. H. Keitel, Under-the-Barrier Dynamics in LaserInduced Relativistic Tunneling, Phys. Rev. Lett. 110, 153004 (2013). 
[31] P. Eckle, M. Smolarski, P. Schlup, J. Biegert, A. Staudte, M. Schöffler, H. G. Muller, R. Dörner, and U. Keller, Attosecond angular streaking, Nat. Phys. 4, 565 (2008).

[32] P. Eckle, A. N. Pfeiffer, C. Cirelli, A. Staudte, R. Dörner, H. G. Muller, M. Büttiker, and U. Keller, Attosecond ionization and tunneling delay time measurements in helium, Science 322, 1525 (2008).

[33] H. Ni, U. Saalmann, and J. M. Rost, Tunneling Ionization Time Resolved by Backpropagation, Phys. Rev. Lett. 117, 023002 (2016).

[34] H. Ni, U. Saalmann, and J. M. Rost, Tunneling exit characteristics from classical backpropagation of an ionized electron wave packet, Phys. Rev. A 97, 013426 (2018).

[35] H. Ni, N. Eicke, C. Ruiz, J. Cai, F. Oppermann, N. I. Shvetsov-Shilovski, and L. W. Pi, Tunneling criteria and a nonadiabatic term for strong-field ionization, Phys. Rev. A 98, 013411 (2018).

[36] C. Hofmann, A. S. Landsman, and U. Keller, Attoclock revisited on electron tunnelling time, J. Mod. Opt. 66, 1052 (2019).

[37] A. S. Kheifets, The attoclock and the tunneling time debate, J. Phys. B 53, 072001 (2020).

[38] S. Brennecke and M. Lein, High-order above-threshold ionization beyond the electric dipole approximation, J. Phys. B 51, 094005 (2018).

[39] X. M. Tong and C. D. Lin, Empirical formula for static field ionization rates of atoms and molecules by lasers in the barrier-suppression regime, J. Phys. B 38, 2593 (2005).

[40] See Supplemental Material at http://link.aps.org/ supplemental/10.1103/PhysRevLett.125.073202 for details, which includes Refs. [41-52].

[41] A. D. Bandrauk and H. Shen, Exponential split operator methods for solving coupled time-dependent Schrödinger equations, J. Chem. Phys. 99, 1185 (1993).

[42] S. P. Goreslavski and S. V. Popruzhenko, Differential photoelectron distributions in a strong elliptically polarized lowfrequency laser field, Sov. Phys. JETP 83, 661 (1996).

[43] N. I. Shvetsov-Shilovski, S. V. Popruzhenko, and S. P. Goreslavski, Asymmetric emission of rescattered photoelectrons in intense laser fields with elliptical polarization, Laser Phys. 13, 1054 (2003).

[44] S. P. Goreslavski, G. G. Paulus, S. V. Popruzhenko, and N. I. Shvetsov-Shilovski, Coulomb Asymmetry in AboveThreshold Ionization, Phys. Rev. Lett. 93, 233002 (2004).

[45] M. V. Frolov, N. L. Manakov, A. A. Minina, S. V. Popruzhenko, and A. F. Starace, Adiabatic-limit Coulomb factors for photoelectron and high-order-harmonic spectra, Phys. Rev. A 96, 023406 (2017).

[46] A. M. Perelomov, V. S. Popov, and M. V. Terent'ev, Ionization of atoms in an alternating electric field, Sov. Phys. JETP 23, 924 (1966).

[47] A. M. Perelomov, V. S. Popov, and M. V. Terent'ev, Ionization of atoms in an alternating electric field: II, Sov. Phys. JETP 24, 207 (1967).

[48] A. M. Perelomov and V. S. Popov, Ionization of atoms in an alternating electric field: III, Sov. Phys. JETP 25, 336 (1967).

[49] V. D. Mur, S. V. Popruzhenko, and V. S. Popov, Energy and momentum spectra of photoelectrons under conditions of ionization by strong laser radiation (The case of elliptic polarization), J. Exp. Theor. Phys. 92, 777 (2001).

[50] V. S. Popov, Tunnel and multiphoton ionization of atoms and ions in a strong laser field (Keldysh theory), Phys. Usp. 47, 855 (2004).

[51] S. V. Popruzhenko, Keldysh theory of strong field ionization: History, applications, difficulties and perspectives, J. Phys. B 47, 204001 (2014).

[52] B. M. Karnakov, V. D. Mur, S. V. Popruzhenko, and V. S. Popov, Current progress in developing the nonlinear ionization theory of atoms and ions, Phys. Usp. 58, 3 (2015).

[53] N. J. Kylstra, R. M. Potvliege, and C. J. Joachain, Photon emission by ions interacting with short intense laser pulses: Beyond the dipole approximation, J. Phys. B 34, L55 (2001).

[54] M. Lein, E. K. U. Gross, and V. Engel, Intense-Field Double Ionization of Helium: Identifying the Mechanism, Phys. Rev. Lett. 85, 4707 (2000).

[55] X. M. Tong, K. Hino, and N. Toshima, Phase-dependent atomic ionization in few-cycle intense laser fields, Phys. Rev. A 74, 031405(R) (2006).

[56] X. M. Tong and S.-I. Chu, Theoretical study of multiple high-order harmonic generation by intense ultrashort pulsed laser fields: A new generalized pseudospectral timedependent method, Chem. Phys. 217, 119 (1997).

[57] X. M. Tong, A three-dimensional time-dependent Schrödinger equation solver: An application to hydrogen atoms in an elliptical laser field, J. Phys. B 50, 144004 (2017).

[58] X. Gao and X. M. Tong, Coulomb effect on the dynamics of atoms in a strong elliptical laser field: Unification of the excitation and ionization, Phys. Rev. A 100, 063424 (2019).

[59] B. Feuerstein and U. Thumm, On the computation of momentum distributions within wavepacket propagation calculations, J. Phys. B 36, 707 (2003).

[60] X. Wang, J. Tian, and J. H. Eberly, Extended Virtual Detector Theory for Strong-Field Atomic Ionization, Phys. Rev. Lett. 110, 243001 (2013).

[61] X. Wang, J. Tian, and J. H. Eberly, Virtual detector theory for strong-field atomic ionization, J. Phys. B 51, 084002 (2018).

[62] R.-H. Xu, X. Wang, and J. H. Eberly, Extended virtual detector theory including quantum interferences, arXiv:2003.05051.

[63] J. P. Wang and F. He, Tunneling ionization of neon atoms carrying different orbital angular momenta in strong laser fields, Phys. Rev. A 95, 043420 (2017).

[64] Q. Zhang, G. Basnayake, A. Winney, Y. F. Lin, D. Debrah, S. K. Lee, and W. Li, Orbital-resolved nonadiabatic tunneling ionization, Phys. Rev. A 96, 023422 (2017).

[65] G. F. Gribakin and M. Y. Kuchiev, Multiphoton detachment of electrons from negative ions, Phys. Rev. A 55, 3760 (1997).

[66] T. K. Kjeldsen and L. B. Madsen, Strong-field ionization of atoms and molecules: The two-term saddle-point method, Phys. Rev. A 74, 023407 (2006).

[67] D. B. Milošević, G. G. Paulus, D. Bauer, and W. Becker, Above-threshold ionization by few-cycle pulses, J. Phys. B 39, R203 (2006).

[68] L. D. Landau and E. M. Lifshits, Course of Theoretical Physics, Vol. 2: The Classical Theory of Fields (Elsevier, Oxford, 1975). 